\title{
CLOSED EMBEDDINGS INTO LIPSCOMB'S UNIVERSAL SPACE
}

\author{
IVAN IVANŠIĆ AND UROŠ MILUTINOVIĆ \\ University of Zagreb, Croatia and University of Maribor, Slovenia \\ Dedicated to Professor Sibe Mardešić on the occasion of his 80th birthday
}

\begin{abstract}
Let $\mathcal{J}(\tau)$ be Lipscomb's one-dimensional space and $L_{n}(\tau)$ $=\left\{x \in \mathcal{J}(\tau)^{n+1} \mid\right.$ at least one coordinate of $x$ is irrational $\} \subseteq \mathcal{J}(\tau)^{n+1}$ Lipscomb's $n$-dimensional universal space of weight $\tau \geq \aleph_{0}$. In this paper we prove that if $X$ is a complete metrizable space and $\operatorname{dim} X \leq n, w X \leq \tau$, then there is a closed embedding of $X$ into $L_{n}(\tau)$. Furthermore, any map $f: X \rightarrow \mathcal{J}(\tau)^{n+1}$ can be approximated arbitrarily close by a closed embedding $\psi: X \rightarrow L_{n}(\tau)$. Also, relative and pointed versions are obtained. In the separable case an analogous result is obtained, in which the classic triangular Sierpiński curve (homeomorphic to $\mathcal{J}(3)$ ) is used instead of $\mathcal{J}\left(\aleph_{0}\right)$.
\end{abstract}

\section{INTRODUCTION AND DEFINITIONS}

If a topological space is embedded into a topologically complete metrizable space (i.e., into a space that can be endowed by a complete metric) as a closed subset, it must be topologically complete metrizable itself. On the other hand, if a topologically complete metrizable space is embedded into another such space, the embedding need not be closed (embedding of $\mathbb{R}$ as an open interval in itself, or $\mathbb{N}$ as $\{1 / m \mid m \in \mathbb{N}\}$ into $\mathbb{R}$, are easy examples for this claim). The problem of the existence of closed embeddings of topologically

2000 Mathematics Subject Classification. 54F45.

Key words and phrases. Covering dimension, embedding, closed embedding, universal space, generalized Sierpiński curve, Lipscomb's universal space, extension, complete metric space.

Work supported in part by the Ministry of Science of Croatia under the Grant P0037105 and by the Ministry of Science of Slovenia under the Grant P1-0297. 
complete metrizable spaces has been extensively treated in the theory of universal spaces. K. Tsuda [15, 16], A. Waśko [17], Y. Hattori [3], W. Olszewski, L. Piąntkiewicz [13], A. Nagórko [12] have proved results about existence of closed embeddings of complete metric spaces into several universal spaces. This often required special modifications of the previously known universal spaces. Also, in all cases the proofs were obtained by the use of the Baire category theorem. For Lipscomb's universal space no results on existence of closed embeddings have appeared yet. In this paper we prove that the direct approach of obtaining embeddings into Lipscomb's universal space, developed in $[9,10]$ and later exploited in $[4,5,6,11]$, yields closed embeddings with no further changes made, in case when the embedded space is topologically complete.

In his papers $[7,8] \mathrm{S}$. L. Lipscomb has defined the space $\mathcal{J}(\tau)$ as a factorspace of Baire's universal 0-dimensional space and used it in his construction of a universal $n$-dimensional metrizable space $L_{n}(\tau)$ of weight $\tau \geq \aleph_{0}$, which is a subspace of $\mathcal{J}(\tau)^{n+1}$. U. Milutinović $[9,10]$ has proved that $\mathcal{J}(\tau)$ is naturally homeomorphic to a generalized Sierpiński curve $\Sigma(\tau)$, which is a subspace of the Hilbert space $\ell_{2}(\tau)$.

Consequently U. Milutinović obtained a geometric realization of $L_{n}(\tau)$. Using such geometric realization, he has proved that $L_{n}(\tau)$ is topologically complete $[9,10]$. J. C. Perry, independently, also proved that $\mathcal{J}(\tau)$ is topologically complete - see [14, p. 2480, footnote]. I. Ivanšić and U. Milutinović used the method developed in $[9,10]$ to prove relative [5] and pointed [6] versions of Lipscomb's universality theorem. In [4] they proved that the classic (fractal) Sierpiński triangle may be used instead of $\mathcal{J}\left(\aleph_{0}\right)$ in the separable case. In [11] U. Milutinović proved that any mapping into $\mathcal{J}(\tau)^{n+1}$, defined on an arbitrary $n$-dimensional metrizable space of weight $\tau \geq \aleph_{0}$, can be approximated by an embedding into $L_{n}(\tau)$.

In this paper we show that any $n$-dimensional complete metric space of weight $\tau \geq \aleph_{0}$ can be embedded into $L_{n}(\tau)$ as a closed subset. We also prove relative and pointed versions of this result, as well as a result about approximations of arbitrary maps by closed embeddings, and a result about the (separable case) universal space based on the Sierpiński triangle.

We shall use the notation of $[1,7]$ (with a few slight modifications).

By dimension we understand the covering dimension.

For the sake of completeness we include here the descriptions of Lipscomb's space $\mathcal{J}(\tau)$, of the generalized Sierpiński curve $\Sigma(\tau)$, and of the homeomorphism between them.

Baire's universal 0-dimensional space of weight $\tau$ is the set $\Lambda^{\mathbb{N}}$ (where $\Lambda$ is a set of cardinality $\tau$ and $\mathbb{N}=\{1,2,3, \ldots\}$ ) equipped with the product topology, while $\Lambda$ is equipped with the discrete topology. Lipscomb's space $\mathcal{J}(\tau)$ is defined as the quotient space $\mathcal{J}(\tau)=\Lambda^{\mathbb{N}} / \sim$, where the equivalence relation $\sim$ is defined as follows: 
for $\lambda=\left(\lambda_{1}, \ldots, \lambda_{m}, \ldots\right), \mu=\left(\mu_{1}, \ldots, \mu_{m}, \ldots\right)$

$\lambda \sim \mu \Longleftrightarrow \lambda=\mu$ or $\exists j \in \mathbb{N}$ such that:

i) $\forall k, k<j \Longrightarrow \lambda_{k}=\mu_{k}$,

ii) $\forall s \in \mathbb{N}, \lambda_{j}=\mu_{j+s}$,

iii) $\forall s \in \mathbb{N}, \lambda_{j+s}=\mu_{j}$.

In the case $\mu \neq \lambda$ such a $j$ is uniquely determined and is called the tail index of $\lambda$ and $\mu$. We also say that the two sequences are interwoven.

The equivalence class of $\left(\lambda_{1}, \ldots, \lambda_{m}, \ldots\right)$ is denoted by $\left[\lambda_{1}, \ldots, \lambda_{m}, \ldots\right]$. An equivalence class may be a singleton - in which case it is called an irrational point of $\mathcal{J}(\tau)$ - or a dyad - in which case it is called a rational point of $\mathcal{J}(\tau)$. $\mathcal{J}(\tau)$ is a one-dimensional metrizable space of weight $\tau[7]$.

The classical Sierpiński triangle may be described as a (fractal) subset of $\mathbb{R}^{3}$ as follows:

Let $e^{1}=(1,0,0), e^{2}=(0,1,0), e^{3}=(0,0,1)$. Let $\varphi_{1}, \varphi_{2}, \varphi_{3}: \mathbb{R}^{3} \rightarrow$ $\mathbb{R}^{3}$ be the homotheties with the coefficients $1 / 2$ and the centers $e^{1}, e^{2}, e^{3}$, respectively. If the convex hull of these three points (i.e., the standard 2simplex) is denoted by $\Sigma$ it is obvious that the set obtained from $\Sigma$ by $k$ removals of the middle triangles may be described as

$$
\Sigma_{k}=\bigcup_{\left(\lambda_{1}, \ldots, \lambda_{k}\right) \in \Lambda^{k}} \varphi_{\lambda_{1}} \circ \cdots \circ \varphi_{\lambda_{k}} \Sigma,
$$

where $\Lambda=\{1,2,3\}$. After that, the Sierpiński triangle is obtained as the intersection of all sets $\Sigma_{k}$.

The generalized Sierpiński curve $\Sigma(\tau)$ is defined analogously using the Hilbert space $\ell_{2}(\tau)=\left\{\left(x_{\lambda}\right) \in \mathbb{R}^{\Lambda} \mid \sum_{\lambda \in \Lambda} x_{\lambda}^{2}<\infty\right\}$ as the ambient space instead of $\mathbb{R}^{3}$. Using $e^{\lambda}, \lambda \in \Lambda$, defined by $\forall \mu \in \Lambda, e_{\mu}^{\lambda}=\delta_{\lambda, \mu}$ (Kronecker's symbol) we describe the "homotheties" with the centers $e^{\lambda}$ and the coefficients $1 / 2$, i.e. the functions $\varphi_{\lambda}: \ell_{2}(\tau) \longrightarrow \ell_{2}(\tau)$ defined by

$$
\left(\varphi_{\lambda}(x)\right)_{\mu}= \begin{cases}\left(x_{\lambda}+1\right) / 2, & \mu=\lambda \\ x_{\mu} / 2, & \mu \neq \lambda .\end{cases}
$$

Let $\sigma=\left\{\left(x_{\lambda}\right) \in \ell_{2}(\tau) \mid \sum_{\lambda \in \Lambda} x_{\lambda}=1 \& \forall \lambda, 0 \leq x_{\lambda} \leq 1\right\}$. Then $\Sigma=\mathrm{Cl} \sigma=$ $\left\{\left(x_{\lambda}\right) \in \ell_{2}(\tau) \mid \sum_{\lambda \in \Lambda} x_{\lambda} \leq 1 \& \forall \lambda, 0 \leq x_{\lambda} \leq 1\right\}$ is the closed convex hull of the set $\left\{e^{\lambda} \mid \lambda \in \Lambda\right\}$ and it may be called the standard $\tau$-simplex by an analogy to the standard $m$-simplex. Now the generalized Sierpiński curve $\Sigma(\tau)$ may be described in the same way as previously the classic curve: subspaces $\Sigma_{k}$ of $\ell_{2}(\tau)$ are defined by (1.1), and then $\Sigma(\tau)$ is defined as

$$
\Sigma(\tau)=\bigcap_{k \in \mathbb{N}} \Sigma_{k}
$$

The points $\varphi_{\lambda_{1}} \circ \cdots \circ \varphi_{\lambda_{k}}\left(e^{\lambda}\right), k \geq 1,\left(\lambda_{1}, \ldots, \lambda_{k}\right) \neq(\lambda, \ldots, \lambda)$, are called the rational points of $\Sigma(\tau)$ (more precisely, for a fixed $k$ they are called the $k$ th 
level vertices), and all other points (including all $e^{\lambda} \mathrm{s}$ ) are irrational points of $\Sigma(\tau)$.

That $\chi: \mathcal{J}(\tau) \longrightarrow \Sigma(\tau)$, defined by

$$
\left\{\chi\left(\left[\lambda_{1}, \ldots, \lambda_{k}, \ldots\right]\right)\right\}=\bigcap_{k \in \mathbb{N}} \varphi_{\lambda_{1}} \circ \cdots \circ \varphi_{\lambda_{k}} \Sigma
$$

is a homeomorphism mapping rational points to rational points and irrational points to irrational points has been proved in [9], and we identify the spaces $\mathcal{J}(\tau)$ and $\Sigma(\tau)$ via $\chi$ freely, choosing the description that is more convenient for use in the context.

Every point of $\Sigma(\tau)$ is thus described by a unique equivalence class of indices $\left[\lambda_{1}, \ldots, \lambda_{k}, \ldots\right]$, where the $\lambda_{k} \mathrm{~s}$ are the indices of the homotheties from (1.2). Any rational point is represented by two interwoven sequences, while any irrational point is represented by a unique sequence. In analogy with decimal expansion of reals, we call the elements $\lambda_{k}$ ciphers of $\left[\lambda_{1}, \ldots, \lambda_{k}, \ldots\right]$.

Let $\mathcal{U}$ be a family of subsets of $X, x \in X$. The local order of $\mathcal{U}$ at $x$ is defined as $\operatorname{lord}_{x} \mathcal{U}=\inf \{k \mid x$ has a neighborhood intersecting $k$ elements of $\mathcal{U}\} \in\{0,1,2, \ldots, \infty\}$. The local order of $\mathcal{U}$ is defined as $\operatorname{lord} \mathcal{U}=\sup \left\{\operatorname{lord}_{x} \mathcal{U} \mid x \in X\right\}$.

$\operatorname{Bd} \mathcal{U}=\bigcup_{U \in \mathcal{U}} \operatorname{Bd} U$, where $\operatorname{Bd} U$ denotes the boundary of the set $U$; $\mathrm{Cl} \mathcal{U}=\bigcup_{U \in \mathcal{U}} \mathrm{Cl} U$, where $\mathrm{Cl} U$ denotes the closure of the set $U$.

A decomposition of the space $X$ is a pairwise disjoint locally finite family of open nonempty subsets of $X$ whose closures cover $X$.

\section{INDEXING OF DECOMPOSITIONS METHOD}

No details of the proofs of theorems in this sections are given, since they have appeared in [9] and [4].

In all mentioned papers of Ivanšić and Milutinović, the main tool used in constructions of embeddings was the following theorem:

THEOREM 2.1. Let $X$ be an $n$-dimensional metrizable space of weight $\tau \geq \aleph_{0}$. Let $X_{1}, \ldots, X_{n+1}$ be fixed pairwise disjoint 0 -dimensional subsets of $X$, such that $X=X_{1} \cup \cdots \cup X_{n+1}$. Then there are decompositions $\mathcal{V}_{i, j}$, $\mathcal{W}_{i, j}$ of $X$, and families $\mathcal{F}_{i, j}$ of pairwise disjoint closed nonempty sets, $i \geq 0$, $1 \leq j \leq n+1$, satisfying the following properties:

(D1) $\mathcal{V}_{i, j}, \mathcal{W}_{i, j}$ are decompositions and lord $\mathcal{V}_{i, j} \leq 2$, lord $\mathcal{W}_{i, j} \leq 2$.

(D2) $x \in \operatorname{Bd} \mathcal{W}_{i, j} \Longleftrightarrow$ there exist distinct members $W_{1}, W_{2}$ of $\mathcal{W}_{i, j}$, such that $x \in \operatorname{Bd} W_{1} \cap \operatorname{Bd} W_{2}$.

(D3) $\mathcal{W}_{i, j}=\mathcal{W}_{i, j}^{S} \cup \mathcal{W}_{i, j}^{B} \cup \mathcal{W}_{i, j}^{R}$, where $\mathcal{W}_{i, j}^{S}, \mathcal{W}_{i, j}^{B}, \mathcal{W}_{i, j}^{R}$ are discrete families which are disjoint in pairs, and $\mathcal{W}_{i, j}^{S} \cup \mathcal{W}_{i, j}^{B}$ is a discrete family (superscripts $S, B, R$ come from small, boundary and remnant, and those are what we call the elements of the families - the terminology is motivated by their properties). 
(D4) $\operatorname{Bd} \mathcal{W}_{i, j} \cap \operatorname{Bd} \mathcal{V}_{i, j}=\emptyset$.

(D5) $\mathcal{W}_{i, j}^{S}$ refines $\mathcal{V}_{i, j}$, i.e., every member of $\mathcal{W}_{i, j}^{S}$ is a subset of an element of $\mathcal{V}_{i, j}$.

(D6) $\mathcal{W}_{i, j}^{R}=\left\{V \backslash \mathrm{Cl}\left(\mathcal{W}_{i, j}^{S} \cup \mathcal{W}_{i, j}^{B}\right) \mid V \in \mathcal{V}_{i, j}, V \backslash \mathrm{Cl}\left(\mathcal{W}_{i, j}^{S} \cup \mathcal{W}_{i, j}^{B}\right) \neq \emptyset\right\}$

(D7) $\mathcal{F}_{i, j}=\left\{\operatorname{Bd} V_{1} \cap \operatorname{Bd} V_{2} \mid V_{1}, V_{2} \in \mathcal{V}_{i, j}, V_{1} \neq V_{2}, \operatorname{Bd} V_{1} \cap \operatorname{Bd} V_{2} \neq \emptyset\right\}$.

(D8) For each $F=\operatorname{Bd} V_{1} \cap \operatorname{Bd} V_{2} \in \mathcal{F}_{i, j}$ there is an element $W \in \mathcal{W}_{i, j}^{B}$, such that $F \subseteq W \subseteq \mathrm{Cl} W \subseteq V_{1} \cup F \cup V_{2}$ (since it is uniquely determined by $F$, we denote it $\left.W_{F}\right) ; \mathcal{W}_{i, j}^{B}=\left\{W_{F} \mid F \in \mathcal{F}_{i, j}\right\}$.

(D9) $\mathcal{V}_{i+1, j}=\left\{V \cap W \mid V \in \mathcal{V}_{i, j}, W \in \mathcal{W}_{i, j}, V \cap W \neq \emptyset\right\}$.

(D10) All the families are of cardinality $\leq \tau$.

(D11) The intersection of the elements from $\mathcal{W}_{k, j}^{B}, k \geq i$, containing a fixed $F \in \mathcal{F}_{i, j}$, is $F$.

(D12) $\operatorname{Bd} \mathcal{W}_{i, j} \cap X_{j}=\emptyset$.

(D13) $\bigcup_{j=1}^{n+1} \mathcal{W}_{i, j}^{S}$ covers $X$, for all $i \geq 0$.

(D14) $\operatorname{diam} W<1 /(i+1)$, for all $W \in \mathcal{W}_{i, j}^{S}$, all $i \geq 0$, and all $j, 1 \leq j \leq$ $n+1$.

REMARK 2.2. It is important to note, that Theorem 2.1 yields all of $\mathcal{V}_{i, j}$, $\mathcal{W}_{i, j}, \mathcal{F}_{i, j}$, starting from arbitrary families $\mathcal{V}_{0, j}, \mathcal{F}_{0, j}, j=1, \ldots, n+1$, that satisfy those properties (D1)-(D14) that apply to them.

Starting from $\mathcal{V}_{0, j}, \mathcal{F}_{0, j}$ we construct $\mathcal{V}_{i, j}, \mathcal{W}_{i, j}, \mathcal{F}_{i, j}$ inductively, as in the following scheme

$$
\mathcal{V}_{0, j}, \mathcal{F}_{0, j} \rightsquigarrow \mathcal{W}_{0, j} \rightsquigarrow \mathcal{V}_{1, j}, \mathcal{F}_{1, j} \rightsquigarrow \mathcal{W}_{1, j} \rightsquigarrow \mathcal{V}_{2, j}, \mathcal{F}_{2, j} \rightsquigarrow \ldots
$$

where all steps of the form $\mathcal{V}_{i, j}, \mathcal{F}_{i, j} \rightsquigarrow \mathcal{W}_{i, j}$ are realized by applications of Lipscomb's lemma [8, Lemma 4, p. 152], while all steps $\mathcal{W}_{i, j} \rightsquigarrow \mathcal{V}_{i+1, j}, \mathcal{F}_{i+1, j}$ are done according to (D7) and (D9).

Also, let us point out that the notation is slightly changed in comparison with [9], due to the change of emphasis from the sets $\mathcal{W}_{i, j}$ to the sets $\mathcal{V}_{i, j}$ that happened meanwhile. For example, in [9] the members of the families $\mathcal{W}_{i, j}$ have been indexed, while in all other papers the members of the families $\mathcal{V}_{i, j}$ are indexed, as in the following theorem.

THEOREM 2.3. Let $X$ be an $n$-dimensional metrizable space of weight $\tau \geq \aleph_{0}$. Let $X_{1}, \ldots, X_{n+1}$ be fixed pairwise disjoint 0-dimensional subsets of $X$, such that $X=X_{1} \cup \cdots \cup X_{n+1}$. Let $\mathcal{V}_{i, j}, \mathcal{W}_{i, j}$, and $\mathcal{F}_{i, j}, i \geq 0$, $1 \leq j \leq n+1$, be as in Theorem 2.1. Then there is an indexing of the elements of $\mathcal{V}_{i, j}, i \geq 0,1 \leq j \leq n+1$, satisfying the following properties:

(I1) Each member of $\mathcal{V}_{i, j}, i \geq 0$, is indexed by an element of $\Lambda^{i+1}$.

(I2) For a given $F \in \bigcup_{i=0}^{\infty} \mathcal{F}_{i, j}$, let $i$ be the least index such that $F \in \mathcal{F}_{i, j}$ (i.e., $F \in \mathcal{F}_{i, j} \backslash \mathcal{F}_{i-1, j},{ }^{1}$ for $i \geq 1$, or $F \in \mathcal{F}_{0, j}$, for $i=0$ ). If

\footnotetext{
${ }^{1}$ It can be shown (i.e., see $\left[4\right.$, Lemma 3]) that $\mathcal{F}_{i-1, j} \subseteq \mathcal{F}_{i, j}$.
} 
$F=\operatorname{Bd} V_{1} \cap \operatorname{Bd} V_{2} \neq \emptyset$, for $V_{1}, V_{2} \in \mathcal{V}_{i, j}, V_{1} \neq V_{2}$, then $V_{1}, V_{2}$ are indexed either by

(A) $\left(\lambda_{1}, \ldots, \lambda_{k}, \mu\right),\left(\lambda_{1}, \ldots, \lambda_{k}, \nu\right), \mu \neq \nu$ (for some $k$ ), or by

(B) $\left(\lambda_{1}, \ldots, \lambda_{k}, \mu, \nu, \ldots, \nu\right),\left(\lambda_{1}, \ldots, \lambda_{k}, \nu, \mu, \ldots, \mu\right), \mu \neq \nu, \quad$ (for some $k$ ).

For any $\ell>i$, let $\tilde{V}_{1}, \tilde{V}_{2} \in \mathcal{V}_{\ell, j}$ such that $F=\operatorname{Bd} \tilde{V}_{1} \cap \operatorname{Bd} \tilde{V}_{2} \cdot{ }^{2}$ Suppose $\tilde{V}_{1} \subseteq V_{\left(\lambda_{1}, \ldots, \lambda_{k}, \mu\right)}$ and $\tilde{V}_{2} \subseteq V_{\left(\lambda_{1}, \ldots, \lambda_{k}, \nu\right)}$ (in case $(\mathrm{A})$ ) or $\tilde{V}_{1} \subseteq$ $V_{\left(\lambda_{1}, \ldots, \lambda_{k}, \mu, \nu, \ldots, \nu\right)}$ and $\tilde{V}_{2} \subseteq V_{\left(\lambda_{1}, \ldots, \lambda_{k}, \nu, \mu, \ldots, \mu\right)}$ (in case $\left.(\mathrm{B})\right) .{ }^{3}$ Then $\tilde{V}_{1}$ is indexed by the $(\ell+1)$-tuple $\left(\lambda_{1}, \ldots, \lambda_{k}, \mu, \nu, \ldots, \nu\right) \in \Lambda^{\ell+1}$, and similarly $\tilde{V}_{2}$ is indexed by the interwoven element $\left(\lambda_{1}, \ldots, \lambda_{k}, \nu, \mu, \ldots, \mu\right) \in$ $\Lambda^{\ell+1}$.

(I3) If $V \in \mathcal{V}_{i, j}, i \geq 0$, is indexed by an index having two or more identical ciphers at the end, then there is an $F \in \mathcal{F}_{\ell, j}, \ell \leq i$, such that $F=$ $\operatorname{Bd} V_{1} \cap \operatorname{Bd} V_{2} \neq \emptyset, V_{1}, V_{2} \in \mathcal{V}_{i, j}, V_{1} \neq V_{2}$, and either $V=V_{1}$ or $V=V_{2}$.

(I4) If $V \in \mathcal{V}_{i, j}, i \geq 0$, is indexed by $\left(\lambda_{1}, \ldots, \lambda_{i+1}\right)$, and if $V^{\prime} \in \mathcal{V}_{k, j}, k>i$, is indexed by $\left(\mu_{1}, \ldots, \mu_{k+1}\right)$, then $V^{\prime} \subseteq V$ implies $\left(\lambda_{1}, \ldots, \lambda_{i+1}\right)=$ $\left(\mu_{1}, \ldots, \mu_{i+1}\right)$.

Now, in order to get an embedding $\psi: X \rightarrow L_{n}(\tau) \subseteq \mathcal{J}(\tau)^{n+1}$, we define the coordinate functions

$$
\psi_{j}: X \longrightarrow \mathcal{J}(\tau)=\Sigma(\tau), \quad j=1, \ldots, n+1,
$$

in the same way as in [9] and [4], i.e., by the formulas

$$
x \longmapsto\left[\lambda_{1}, \ldots, \lambda_{k}, \mu, \nu, \ldots, \nu, \ldots\right]=\left[\lambda_{1}, \ldots, \lambda_{k}, \nu, \mu, \ldots, \mu, \ldots\right],
$$

when $x$ belongs to the boundaries of the sets in $\mathcal{V}_{i, j}, i \geq 0$, indexed by the initial segments of the sequences $\lambda_{1}, \ldots, \lambda_{k}, \mu, \nu, \ldots, \nu, \ldots$ and $\lambda_{1}, \ldots, \lambda_{k}, \nu$, $\mu, \ldots, \mu, \ldots$, or by

$$
x \longmapsto\left[\lambda_{1}, \ldots, \lambda_{k}, \ldots\right],
$$

when $x$ belongs to the sets in $\mathcal{V}_{i, j}$, for all $i \geq 0$, indexed by the initial segments of the sequence $\lambda_{1}, \ldots, \lambda_{k}, \ldots$ and belongs to no boundary of elements of the decompositions $\mathcal{V}_{i, j}$.

This means, if $x \in F$, where $F \in \mathcal{F}_{i, j}$, and $i$ is the least index with this property (cf. (I2)), where $F=\operatorname{Bd} V_{1} \cap \mathrm{Bd} V_{2} \neq \emptyset, V_{1}, V_{2} \in \mathcal{V}_{i, j}, V_{1} \neq V_{2}$, and $V_{1}$ and $V_{2}$ are indexed according to (I2), then $\psi_{j}(x)$ is defined to be

$$
\psi_{j}(x)=\left[\lambda_{1}, \ldots, \lambda_{k}, \mu, \nu, \ldots, \nu, \ldots\right]=\left[\lambda_{1}, \ldots, \lambda_{k}, \nu, \mu, \ldots, \mu, \ldots\right] .
$$

Hence, in this case, $\psi_{j}(x)$ is a rational point of $\Sigma(\tau)$.

\footnotetext{
${ }^{2}$ We know that $F$ must be of this form, by (D7) and by the previous footnote.

${ }^{3}$ From the obvious fact that $\mathcal{V}_{i, j}$ refines $\mathcal{V}_{i-1, j}$, it follows that always either this is the case, or that the formulas obtained from these by interchanging $\tilde{V}_{1}, \tilde{V}_{2}$ hold true.
} 
If $x$ does not belong to any $F \in \bigcup_{i=0}^{\infty} \mathcal{F}_{i, j}$, then there is a unique sequence $\lambda_{1}, \lambda_{2}, \ldots$, such that for any $i \geq 0$

$$
x \in V_{\left(\lambda_{1}, \ldots, \lambda_{i+1}\right)} \in \mathcal{V}_{i, j} .
$$

Then we define

$$
\psi_{j}(x)=\left[\lambda_{1}, \ldots, \lambda_{k}, \ldots\right] .
$$

Because of (I3) and (D11), $\psi_{j}(x)$ is an irrational point of $\Sigma(\tau)$.

In [9] and [4] it was proved (by proving that $\psi=\left(\psi_{1}, \ldots, \psi_{n+1}\right)$ is continuous, that the family $\left\{\psi_{1}, \ldots, \psi_{n+1}\right\}$ separates points and closed sets, and that $\left.\psi(X) \subseteq L_{n}(\tau) \subseteq \Sigma(\tau)^{n+1}\right)$ that the following theorem holds true:

THEOREM 2.4. Under previous assumptions and meaning of notation

$$
\psi=\left(\psi_{1}, \ldots, \psi_{n+1}\right): X \longrightarrow L_{n}(\tau)
$$

is an embedding.

\section{Closed embeddability of complete spaces}

In this section we prove the main result of the paper (Theorem 3.3), but we first characterize those embeddings of complete metric spaces which are closed.

Proposition 3.1. Let $f: X \longrightarrow Y$ be an embedding of a complete metric space $X$ into a metric space $Y$. Then $f(X)$ is closed in $Y$ if and only if for any sequence $\left(x_{k}\right)$ in $X$, the convergence of $\left(f\left(x_{k}\right)\right)$ in $Y$ implies $\left(x_{k}\right)$ is Cauchy in $X$.

Proof. If $f(X)$ is closed and if $\left(f\left(x_{k}\right)\right)$ is convergent in $Y$, then $\left(f\left(x_{k}\right)\right)$ converges to $f(x)$, for some $x \in X$. Then, by continuity of $f^{-1}$ on $f(X),\left(x_{k}\right)$ converges to $x$, hence $\left(x_{k}\right)$ is Cauchy.

Conversely, let $y \in \operatorname{Cl} f(X)$ be an arbitrary point. Let $\left(x_{k}\right)$ be a sequence in $X$, such that $\left(f\left(x_{k}\right)\right)$ converges to $y$. Then $\left(x_{k}\right)$ is Cauchy, hence it is convergent, and let $x$ be its limit. By the continuity of $f$, it follows that $\left(f\left(x_{k}\right)\right)$ converges to $f(x)$. Therefore $y=f(x) \in f(X)$. This proves $\mathrm{Cl} f(X) \subseteq f(X)$; hence $\mathrm{Cl} f(X)=f(X)$, and $f(X)$ is closed.

Another key ingredient of the proof of Theorem 3.3 will be the following lemma.

Lemma 3.2. The mapping $\psi_{j}: X \rightarrow \Sigma(\tau)$, defined by (2.1) and (2.2), satisfies

$$
x \in V_{\left(\lambda_{1}, \ldots, \lambda_{m}\right)} \Longrightarrow \psi_{j}(x) \in \varphi_{\lambda_{1}} \circ \cdots \circ \varphi_{\lambda_{m}} \Sigma,
$$

for any $x \in X$ and for any $m \in \mathbb{N}$. 
Proof. If $x \in F \in \bigcup_{i=0}^{\infty} \mathcal{F}_{i, j}$, let $i$ be the least index with the property $F \in \mathcal{F}_{i, j}$. Then, by (D7), $F$ is of the form $F=\operatorname{Bd} V_{1} \cap \operatorname{Bd} V_{2} \neq \emptyset, V_{1}, V_{2} \in \mathcal{V}_{i, j}$, $V_{1} \neq V_{2}$.

By $(2.1), \psi_{j}(x)=\left[\pi_{1}, \pi_{2}, \ldots\right]=\left[\rho_{1}, \rho_{2}, \ldots\right]$, where $\pi_{1}, \pi_{2}, \ldots$ and $\rho_{1}, \rho_{2}, \ldots$ are two interwoven sequences, and $V_{1}, V_{2}$ are indexed by $\left(\pi_{1}, \ldots, \pi_{i+1}\right)$, $\left(\rho_{1}, \ldots, \rho_{i+1}\right)$ (of course, the indices satisfy (A) or (B) of (I2)).

Then $m<i$, because $x$ is in the open set $V_{\left(\lambda_{1}, \ldots, \lambda_{m}\right)}$ and $x \in F \in \mathcal{F}_{i, j} \subseteq$ $\mathcal{F}_{i+1, j} \subseteq \mathcal{F}_{i+2, j} \subseteq \cdots$ and because of (D7) (see footnote 1 and the definition of a decomposition).

Let $V \in \mathcal{V}_{m-1, j}$ be such that $x \in V$. From Theorem 2.1 it follows that $V_{1}, V_{2} \subseteq V$ ((D9), (D3), (D8)), and from (I4) it follows that $\left(\lambda_{1}, \ldots, \lambda_{m}\right)=$ $\left(\pi_{1}, \ldots, \pi_{m}\right)$ and $\left(\lambda_{1}, \ldots, \lambda_{m}\right)=\left(\rho_{1}, \ldots, \rho_{m}\right)$. But then, by $(2.1)$, taking into account (1.2),

$$
\left\{\psi_{j}(x)\right\}=\bigcap_{k \in \mathbb{N}} \varphi_{\pi_{1}} \circ \cdots \circ \varphi_{\pi_{k}} \Sigma \subseteq \varphi_{\pi_{1}} \circ \cdots \circ \varphi_{\pi_{m}} \Sigma=\varphi_{\lambda_{1}} \circ \cdots \circ \varphi_{\lambda_{m}} \Sigma,
$$

and, redundantly,

$$
\left\{\psi_{j}(x)\right\}=\bigcap_{k \in \mathbb{N}} \varphi_{\rho_{1}} \circ \cdots \circ \varphi_{\rho_{k}} \Sigma \subseteq \varphi_{\rho_{1}} \circ \cdots \circ \varphi_{\rho_{m}} \Sigma=\varphi_{\lambda_{1}} \circ \cdots \circ \varphi_{\lambda_{m}} \Sigma .
$$

If $x$ does not belong to any $F \in \bigcup_{i=0}^{\infty} \mathcal{F}_{i, j}$, then by (2.3) there is a uniquely determined sequence $\lambda_{1}, \lambda_{2}, \ldots$, such that $x \in V_{\left(\lambda_{1}, \ldots, \lambda_{i}\right)}$, for all $i \in \mathbb{N}$, and by (2.2), taking into account (1.2), we get

$$
\psi_{j}(x) \in \varphi_{\lambda_{1}} \circ \cdots \circ \varphi_{\lambda_{m}} \Sigma \text {. }
$$

THEOREM 3.3. Let $(X, \mathrm{~d})$ be an $n$-dimensional complete metric space of weight $\tau \geq \aleph_{0}$. Then there is a closed embedding of $X$ into $L_{n}(\tau)$.

Proof. Applying Theorems 2.1 and 2.3 and Remark 2.2 to $(X, \mathrm{~d})$, and to fixed pairwise disjoint 0 -dimensional subsets $X_{1}, \ldots, X_{n+1}$ of $X$, such that $X=X_{1} \cup \ldots \cup X_{n+1}$, and starting from $\mathcal{V}_{0, j}=\{X\}, \mathcal{F}_{0, j}=\emptyset$, one gets families $\mathcal{V}_{i, j}, \mathcal{W}_{i, j}, \mathcal{F}_{i, j}, i \geq 0, j=1, \ldots, n+1$, satisfying (D1)-(D14), and indexing of the elements of $\mathcal{V}_{i, j}, i \geq 0, j=1, \ldots, n+1$, satisfying (I1)-(I4).

For each $j=1, \ldots, n+1$ let $\psi_{j}: X \rightarrow \Sigma(\tau)$ be defined by (2.1) and (2.2). By Theorem 2.4, $\psi=\left(\psi_{1}, \ldots, \psi_{n+1}\right)$ is an embedding of $X$ into $L_{n}(\tau)$.

As an application of Proposition 3.1 we shall now show that $\psi(X)$ is closed.

STEP 1. Let $\left(x_{k}\right)$ be an arbitrary sequence in $X$, such that $\left(\psi\left(x_{k}\right)\right)$ is convergent in $L_{n}(\tau)$. Let $y=\lim _{k} \psi\left(x_{k}\right) \in L_{n}(\tau) \subseteq \Sigma(\tau)^{n+1}$.

For each $k$ and for any $i$, there is a $j$ such that $x_{k} \in \cup \mathcal{W}_{i, j}^{S}$, by (D13). Then there is a $j$ such that the set $\mathbb{N}_{i, j}=\left\{k \in \mathbb{N} \mid x_{k} \in \bigcup \mathcal{W}_{i, j}^{S}\right\}$ is infinite for infinitely many $i$. If not, for each $j$ there would be an $i_{j}$ such that $i>i_{j}$ 
implies $\mathbb{N}_{i, j}$ is finite. For any $i>\max \left\{i_{1}, \ldots, i_{n+1}\right\}$ the set $\bigcup_{j=1}^{n+1} \mathbb{N}_{i, j}=$ $\left\{k \in \mathbb{N} \mid x_{k} \in \bigcup_{j=1}^{n+1}\left(\bigcup \mathcal{W}_{i j}^{S}\right)\right\}$ would be finite. But this is impossible, since $\bigcup_{j=1}^{n+1}\left(\bigcup \mathcal{W}_{i j}^{S}\right)=X$, by (D13).

Fix a $j$ such that for infinitely many $i$ the set $\mathbb{N}_{i, j}$ is infinite.

STEP 2. First we prove that $y_{j}$ is irrational. If not,

$$
y_{j}=\left[\lambda_{1}, \ldots, \lambda_{k}, \mu, \nu, \ldots, \nu, \ldots\right]=\left[\lambda_{1}, \ldots, \lambda_{k}, \nu, \mu, \ldots, \mu, \ldots\right],
$$

for some $\lambda_{1}, \ldots, \lambda_{k}, \mu, \nu \in \Lambda$. Choose an $m>k+2$, such that $\mathbb{N}_{m, j}$ is infinite. Then

$$
\begin{aligned}
\Omega=\operatorname{Int}(\Sigma(\tau) \cap & \left(\varphi_{\lambda_{1}} \circ \ldots \circ \varphi_{\lambda_{k}} \circ \varphi_{\mu} \circ \varphi_{\nu} \circ \ldots \circ \varphi_{\nu} \Sigma\right. \\
& \left.\left.\cup \varphi_{\lambda_{1}} \circ \ldots \circ \varphi_{\lambda_{k}} \circ \varphi_{\nu} \circ \varphi_{\mu} \circ \ldots \circ \varphi_{\mu} \Sigma\right)\right),
\end{aligned}
$$

where in both cases we have compositions of $m+2$ functions $\varphi$, is an open neighborhood of $y_{j}$. Therefore $\Omega$ contains almost all $\psi_{j}\left(x_{k}\right)$, since $\lim _{k} \psi_{j}\left(x_{k}\right)=y_{j}$. Choose $k$, such that $\psi_{j}\left(x_{k}\right) \in \Omega$, and such that $k \in \mathbb{N}_{m, j}$, i.e., such that $x_{k} \in \bigcup \mathcal{W}_{m, j}^{S}$.

Let $W$ be the member of the family $\mathcal{W}_{m, j}^{S}$ such that $x_{k} \in W$. By (D5) and (D9), $W \in \mathcal{V}_{m+1, j}$, hence $W=V_{\left(\kappa_{1}, \ldots, \kappa_{m+2}\right)}$, for some $\left(\kappa_{1}, \ldots, \kappa_{m+2}\right) \in \Lambda^{m+2}$, by (I1).

Then $\left(\kappa_{1}, \ldots, \kappa_{m+2}\right) \neq\left(\lambda_{1}, \ldots, \lambda_{k}, \mu, \nu, \ldots, \nu\right) \in \Lambda^{m+2}$, by (I3) (no small set can be indexed that way). Analogously

$$
\left(\kappa_{1}, \ldots, \kappa_{m+2}\right) \neq\left(\lambda_{1}, \ldots, \lambda_{k}, \nu, \mu, \ldots, \mu\right) \in \Lambda^{m+2} .
$$

Therefore $\varphi_{\kappa_{1}} \circ \cdots \circ \varphi_{\kappa_{m+2}} \Sigma \cap \Omega=\emptyset$ (for details see [9]). Since, by Lemma 3.2, $\psi_{j}(x) \in \varphi_{\kappa_{1}} \circ \cdots \circ \varphi_{\kappa_{m+2}} \Sigma$, it follows that $\psi_{j}\left(x_{k}\right) \notin \Omega$, yielding a contradiction.

Hence, $y_{j}$ is irrational, and therefore there is a unique sequence $\lambda_{1}, \lambda_{2}, \ldots$ $\in \Lambda$, such that $y_{j}=\left[\lambda_{1}, \lambda_{2}, \ldots\right]$.

STEP 3. Let $\varepsilon>0$ be arbitrary. Choose $m$, such that $1 /(m+1)<\varepsilon$ and such that $\mathbb{N}_{m, j}$ is infinite, i.e., such that $x_{k} \in \bigcup \mathcal{W}_{m, j}^{S}$ for infinitely many $k$.

Now, $\Omega^{\prime}=\operatorname{Int}\left(\Sigma(\tau) \cap\left(\varphi_{\lambda_{1}} \circ \varphi_{\lambda_{2}} \circ \ldots \circ \varphi_{\lambda_{m+2}} \Sigma\right)\right)$ is an open neighborhood of $y_{j}$. Choose $k_{0}$, such that $\psi_{j}\left(x_{k}\right) \in \Omega^{\prime}$ for all $k \geq k_{0}$. Choose $k$, such that $k \geq k_{0}$ and that $k \in \mathbb{N}_{m, j}$, i.e., such that $x_{k} \in \bigcup \mathcal{W}_{m, j}^{S}$.

If $x_{k} \in \mathcal{F}_{m+1, j}$, by (D8) and (I1), $F=\operatorname{Bd} V_{\left(\mu_{1}, \ldots, \mu_{m+2}\right)} \cap \mathrm{Bd} V_{\left(\nu_{1}, \ldots, \nu_{m+2}\right)}$, for some $\mu_{1}, \ldots, \mu_{m+2}, \nu_{1}, \ldots, \nu_{m+2} \in \Lambda$. From (1.2) and (2.1) it follows that

$$
\psi_{j}\left(x_{k}\right) \in \varphi_{\mu_{1}} \circ \cdots \circ \varphi_{\mu_{m+2}} \Sigma \cap \varphi_{\nu_{1}} \circ \cdots \circ \varphi_{\nu_{m+2}} \Sigma,
$$

and since $\left(\mu_{1}, \ldots, \mu_{m+2}\right) \neq\left(\nu_{1}, \ldots, \nu_{m+2}\right)$, it follows that $\psi_{j}\left(x_{k}\right)$ is a $(m+2)$ nd level vertex. Since $\Omega^{\prime}$ contains no such vertex [9], it follows that $x \in$ $\cup \mathcal{V}_{m+1, j}$ (recall that $\mathcal{V}_{m+1, j}$ is a decomposition of $X$ ).

Therefore (by Lemma 3.2) $x_{k} \in V_{\left(\lambda_{1}, \ldots, \lambda_{m+2}\right)}$ (all other $\varphi_{\kappa_{1}} \circ \cdots \circ \varphi_{\kappa_{m+2}} \Sigma$ are disjoint with $\Omega^{\prime}$ ). Let $W \in \mathcal{W}_{m, j}^{S}$, such that $x_{k} \in W$. Since $\mathcal{W}_{m, j}^{S} \subseteq \mathcal{V}_{m+1, j}$, 
and since $\mathcal{V}_{m+1, j}$ is a decomposition of $X$, it follows that $V_{\left(\lambda_{1}, \ldots, \lambda_{m+2}\right)}=W \in$ $\mathcal{W}_{m, j}^{S}$. Hence $\operatorname{diam} V_{\left(\lambda_{1}, \ldots, \lambda_{m+2}\right)}<1 /(m+1)<\varepsilon$, by (D14).

For any $p, q \geq k_{0}$, we have $x_{p}, x_{q} \in V_{\left(\lambda_{1}, \ldots, \lambda_{m+2}\right)}$ (the proof is similar as for $x_{k}$ above), hence $\mathrm{d}\left(x_{p}, x_{q}\right)<\varepsilon$, and it means that $x_{k}$ is a Cauchy sequence.

\section{Approximation By Closed Embeddings}

Let D be the maximum metric on $\Sigma(\tau)^{n+1}$, where the metric on $\Sigma(\tau)$ is induced by the metric on $\ell_{2}(\tau)$.

THEOREM 4.1. Let $(X, \mathrm{~d})$ be an $n$-dimensional complete metric space of weight $w X \leq \tau$, and let $f: X \rightarrow \Sigma(\tau)^{n+1}$ be a continuous map. Then for any $\varepsilon>0$ there is a closed embedding $\psi: X \rightarrow L_{n}(\tau)$ such that $\forall x \in$ $X, \mathrm{D}(f(x), \psi(x)) \leq \varepsilon$.

Proof. We choose the families $\mathcal{V}_{0, j}, \mathcal{F}_{0, j}, j=1, \ldots, n+1$ as in [11] and then continue as in the proof of Theorem 2.4. In [11] it was proved that $\psi: X \rightarrow L_{n}(\tau)$ obtained that way is an embedding satisfying $\forall x \in$ $X, \mathrm{D}(f(x), \psi(x)) \leq \varepsilon$, and the proof of Theorem 3.3 applies in this case too and proves that $\psi$ is closed.

\section{Pointed VERsion}

In [6] it was proved that for any $n$-dimensional metrizable space $X$ of weight $\tau \geq \aleph_{0}$, and for any finite subset $X^{\prime}=\left\{x_{1}, \ldots, x_{m}\right\}$ of $X$, any embedding of $X^{\prime}$ into $L_{n}(\tau)$ can be extended to an embedding of $X$ into $L_{n}(\tau)$. For $n \geq 1$ this was proved by including $X$ into a larger $n$-dimensional space $\tilde{X}$ of weight $\tau$, and then by applying the procedure of Section 2 to $\tilde{X}$. After obtaining an embedding of $\tilde{X}$ into $L_{n}(\tau)$ it was shown that its restriction to $X$ satisfied all required properties.

The space $\tilde{X}$ is defined as follows. Fix a metric d on $X$. Then $\tilde{X}$ is obtained from the disjoint union of $X$ and $[-1,1] \times\{1,2, \ldots, m\}$, by identification of each point $x_{k}$ with $(0, k)$. Define $\tilde{\mathrm{d}}: \tilde{X} \times \tilde{X} \rightarrow \mathbb{R}$ as follows:

$$
\tilde{\mathrm{d}}(a, b)= \begin{cases}\mathrm{d}(a, b) & \text { if } a, b \in X, \\ \mathrm{~d}\left(a, x_{k}\right)+|t| & \text { if } a \in X, b=(t, k) \\ \mathrm{d}\left(x_{k}, b\right)+|t| & \text { if } b \in X, a=(t, k) \\ |s-t| & \text { if } a=(s, k), b=(t, k), \\ |s|+\mathrm{d}\left(x_{k}, x_{\ell}\right)+|t| & \text { if } a=(s, k), b=(t, \ell), k \neq \ell .\end{cases}
$$

It is easily checked that $\tilde{\mathrm{d}}$ is a metric on $\tilde{X}$ extending $\mathrm{d}$ and that the dimension and the weight of $X$ are preserved. See Figure 1.

LEMma 5.1. $[-1,1] \times\{k\}$ is closed in $\tilde{X}$, for $k=1, \ldots, m ; X$ is closed in $\tilde{X}$. 


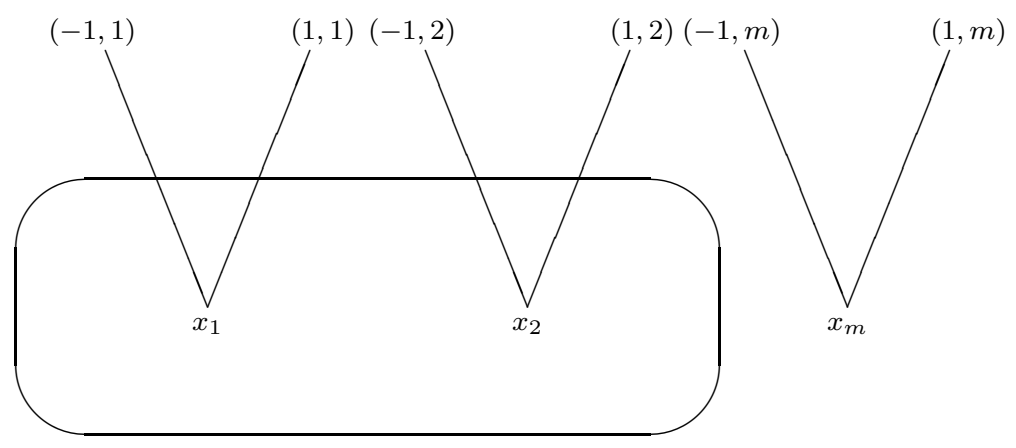

FIGURE 1. $\tilde{X}$ with $x_{m}$ isolated in $X$.

Proof. The first claim follows from the compactness of these sets; the second from $\tilde{X} \backslash X=\bigcup_{k=1}^{m}(B((-1, k), 1) \cup B((1, k), 1))$, where $B(x, r)$ denotes the open ball in $(\tilde{X}, \tilde{\mathrm{d}})$ centered at $x$, of the radius $r$.

Lemma 5.2. If $(X, \mathrm{~d})$ is complete, then $(\tilde{X}, \tilde{\mathrm{d}})$ is complete.

Proof. Let $\left(t_{p}\right)$ be a Cauchy sequence in $(\tilde{X}, \tilde{\mathrm{d}})$.

Since $\tilde{\mathrm{d}}\left(t_{p}, t_{q}\right)=|s|+\mathrm{d}\left(x_{k}, x_{\ell}\right)+|t| \geq \mathrm{d}\left(x_{k}, x_{\ell}\right)>0$ if $t_{p}=(s, k), t_{q}=(t, \ell)$ for $k \neq \ell$, it follows that there are only finitely many such pairs $\left(t_{p}, t_{q}\right)$.

If there is a convergent subsequence of $\left(t_{p}\right)$ in $[-1,1] \times\{k\}$, it has the limit in $[-1,1] \times\{k\}$, since $[-1,1] \times\{k\}$ is closed in $\tilde{X}$, by Lemma 5.1 . The same holds true for convergent subsequences in $X$.

From compactness of $[-1,1] \times\{k\}$, from completeness of $(X, \mathrm{~d})$, and from the fact that a Cauchy sequence having a convergent subsequence is itself convergent, it follows that there are only the following three cases:

1. Almost all $t_{p}$ are in $X$. In this case $\left(t_{p}\right)$ converges to an element of $X$ (we use the completeness of $(X, \mathrm{~d})$ ).

2. Almost all $t_{p}$ are in $[-1,1] \times\{k\}$, for some $k=1, \ldots, m$. In this case $\left(t_{p}\right)$ converges to an element of $[-1,1] \times\{k\}$ (we use the compactness of $[-1,1] \times\{k\})$.

3. There are infinitely many $t_{p}$ in $X$, and infinitely many in $[-1,1] \times$ $\{k\}$, for some $k=1, \ldots, m$. In this case $\left(t_{p}\right)$ converges to $x_{k}$ (the subsequence of $\left(t_{p}\right)$, consisting of all $t_{p}$ belonging to $X$, has the limit in $X$, the subsequence of $\left(t_{p}\right)$, consisting of all $t_{p}$ belonging to $[-1,1] \times$ $\{k\}$, has the limit in $[-1,1] \times\{k\}$, both limits are equal, and the only point common to $X$ and $[-1,1] \times\{k\}$ is $x_{k}$ ). 
Now we are able to prove

THEOREM 5.3. Let $X$ be an $n$-dimensional $(n \geq 0)$ complete metrizable space of weight $\tau \geq \aleph_{0}$. Let $\left\{x_{1}, \ldots, x_{m}\right\}$ be any ordered set of $m$ different points of $X$; similarly let $\left\{y_{1}, \ldots, y_{m}\right\}$ be any ordered set of $m$ different points of $L_{n}(\tau)$. Then there is a closed embedding $\psi: X \rightarrow L_{n}(\tau)$ such that $\psi\left(x_{k}\right)=$ $y_{k}$ for any $k=1, \ldots, m$.

Proof. Let $n \geq 1$ (the 0 -dimensional case will follow from the results of the next section).

In [6] it is explained in details how to obtain decompositions $\mathcal{V}_{0, j}$ of $\tilde{X}$ and families $\mathcal{F}_{0, j}$ of closed subsets of $\tilde{X}$ from which the methods of Section 2 give families $\mathcal{V}_{i, j}, \mathcal{W}_{i, j} \mathcal{F}_{i, j}$ and an indexing of families $\mathcal{V}_{i, j}$ satisfying (D1)(D14) (in fact, satisfying also certain additional properties (D15), (D16)) and (I1)-(I4) ${ }^{4}$ (also satisfying certain (I5), (I6)), such that the resulting function $\tilde{\psi}: \tilde{X} \rightarrow L_{n}(\tau)$, satisfying (2.1) and (2.2), is an embedding, and that the restriction $\psi=\tilde{\psi} \mid X: X \rightarrow L_{n}(\tau)$ is an embedding mapping each $x_{k}$ to $y_{k}$, $k=1, \ldots, m$.

The same proof we used in proving Theorem 3.3 shows that $\tilde{\psi}(\tilde{X})$ is closed in $L_{n}(\tau)$. Since $X$ is closed in $\tilde{X}$, by Lemma 5.1 , it follows that $\psi(X)=\tilde{\psi}(X)$ is closed in $\tilde{\psi}(\tilde{X})$, hence it is closed in $L_{n}(\tau)$, too.

\section{Relative 0-Dimensional Version}

In [5] the following theorem was proved:

TheOREM 6.1. Let $X$ be a 0 -dimensional metric space of weight $\tau \geq \aleph_{0}$ and let $X_{0}$ be a compact subspace of $X$. Then any embedding $\psi_{0}: X_{0} \rightarrow L_{0}(\tau)$ can be extended to an embedding $\psi: X \rightarrow L_{0}(\tau)$.

Using the same reasoning as in previous sections, one may prove:

TheOREM 6.2. Let $X$ be a 0 -dimensional complete metric space of weight $\tau \geq \aleph_{0}$ and let $X_{0}$ be a compact subspace of $X$. Then any embedding $\psi_{0}$ : $X_{0} \rightarrow L_{0}(\tau)$ can be extended to a closed embedding $\psi: X \rightarrow L_{0}(\tau)$.

\section{Separable case}

In [4] it has been proved, that

THEOREM 7.1. The subspace

$$
L_{n}(3)=\left\{x \in \Sigma(3)^{n+1} \mid \text { at least one coordinate of } x \text { is irrational }\right\}
$$

of $\Sigma(3)^{n+1}$ is a universal space for the class of all $n$-dimensional separable metrizable spaces.

\footnotetext{
${ }^{4}$ There is a minor difference, having no consequences for the rest of the proof: the indexing set of elements of $\mathcal{V}_{i, j}$ is $\Lambda^{i+p}$, for some $p \geq 1$, instead of $\Lambda^{i+1}$.
} 
Using the methods explained above, one can prove:

TheOREM 7.2. Let $(X, \mathrm{~d})$ be an $n$-dimensional separable complete metric space. Then there is a closed embedding of $X$ into $L_{n}(3)$.

Combining methods of $[4,11]$, and of the present paper, one proves:

ThEOREM 7.3. Let $(X, \mathrm{~d})$ be an $n$-dimensional separable complete metric space, and let $f: X \rightarrow \Sigma(3)^{n+1}$ be a continuous map. Then for any $\varepsilon>0$ there is a closed embedding $\psi: X \rightarrow L_{n}(3)$ such that $\forall x \in X, \mathrm{D}(f(x), \psi(x)) \leq \varepsilon$.

In this case $\mathrm{D}$ is the maximum metric on $\Sigma(3)^{n+1}$, where the metric on $\Sigma(3)$ is induced by the metric on $\mathbb{R}^{3}$.

ACKNOWLEDGEMENT.

Many thanks to the referee for several useful remarks.

\section{REFERENCES}

[1] R. Engelking, Dimension Theory, PWN-Polish Scientific Publishers, Warszawa and North-Holland Publishing Co., Amsterdam-Oxford-New York, 1978.

[2] R. Engelking, General Topology, Revised and completed edition, Heldermann Verlag, Berlin, 1989

[3] Y. Hattori, A note on universal spaces for finite dimensional complete metric spaces, Glas. Mat. Ser. III 24(44) (1989), 139-147.

[4] I. Ivanšić and U. Milutinović, A universal separable metric space based on the triangular Sierpiński curve, Topology Appl. 120 (2002), 237-271.

[5] I. Ivanšić and U. Milutinović, Relative embeddability into Lipscomb's 0-dimensional universal space, Houston J. Math. 29 (2003), 1001-1012.

[6] I. Ivanšić and U. Milutinović, The pointed version of Lipscomb's embedding theorem, Houston J. Math. 31 (2005), 173-192.

[7] S. L. Lipscomb, A universal one-dimensional metric space, in: TOPO 72 - General Topology and its Applications (Proc. Second Pittsburgh Internat. Conf., Pittsburgh, 1972.), Lecture Notes in Math. 378, Springer, Berlin, 1974., 248-257.

[8] S. L. Lipscomb, On imbedding finite-dimensional metric spaces, Trans. Amer. Math. Soc. 211 (1975), 143-160.

[9] U. Milutinović, Completeness of the Lipscomb universal space, Glas. Mat. Ser. III 27(47) (1992), 343-364.

[10] U. Milutinović, Contributions to the theory of universal spaces, Ph. D. Thesis (in Croatian), University of Zagreb, 1993.

[11] U. Milutinović, Approximation of maps into Lipscomb's space by embeddings, Houston J. Math. 32 (2006), 143-159.

[12] A. Nagórko, Characterization and topological rigidity of Nöbeling manifolds, Ph. D. Thesis, Warsaw University, 2006, http://arxiv.org/abs/math/0602574.

[13] W. Olszewski, L. Piąntkiewicz, Closed embeddings of completely metrizable spaces into universal spaces, Glas. Mat. Ser. III 27(47) (1992), 175-181.

[14] J. C. Perry, Lipscomb's universal space is the attractor of an infinite iterated function system, Proc. Amer. Math. Soc. 124 (1996), 2479-2489.

[15] K. Tsuda, A note on closed embeddings of finite-dimensional metric spaces, Bull. London Math. Soc. 17 (1985), 275-278.

[16] K. Tsuda, A note on closed embeddings of finite-dimensional metric spaces II, Bull. Polish Acad. Sci. Math. 33 (1985), 541-546. 
[17] A. Waśko, Spaces universal under closed embeddings for finite-dimensional complete metric spaces, Bull. London Math. Soc. 18 (1986), 293-298.

I. Ivanšić

FER, University of Zagreb

Unska 3, 10000 Zagreb

Croatia

E-mail: ivan.ivansic@fer.hr

U. Milutinović

FNM, University of Maribor

Koroška cesta 160, 2000 Maribor

Slovenia

E-mail: uros.milutinovic@uni-mb.si

Received: 10.6 .2006 .

Revised: 4.7.2006. 